\title{
GYÖRGY LIGETI - FRANCIS BAYER : ENTRETIENS (EXTRAITS)
}

\author{
SIMON GALLOT \\ Chanteur lyrique, directeur artistique de l'ensemble La Note Brève \\ (musique ancienne), auteur d'une thèse sur György Ligeti \\ simon.gallot@hotmail.fr
}

\begin{abstract}
Dans les années quatre-vingt, le compositeur français, Francis Bayer, a réalisé de longues heures d'entretien avec György Ligeti. L'extrait présenté ici concerne la jeunesse de Ligeti, en Transylvanie, et ses premières années de compositeur, pendant la guerre et immédiatement après la guerre, à Budapest. Ligeti y apparaît marqué par l'héritage de Bartók et par l'atmosphère studieuse et exigeante du milieu intellectuel au sein duquel il a été élevé. On apprend également quelques éléments de sa vie liés au destin des Juifs à cette époque.
\end{abstract}

Mots-clefs : György Ligeti, Francis Bayer, Transylvanie, judaïté, intellectualité, création musicale, musique traditionelle

J'ai rencontré Francis Bayer (1938-2004) chez lui à Paris au début des années 2000. C'était pendant mes études universitaires, je préparais ma thèse sur Ligeti. Bayer, quant à lui, venait de prendre sa retraite de Professeur à l'Université Paris 8 . Il était très respecté et apprécié, tant comme musicologue que comme compositeur. J'avais lu (et beaucoup aimé) son ouvrage De Schönberg à Cage, et l'on m'avait conseillé de le rencontrer. Dans les années 1980, il avait en effet entrepris un travail approfondi sur l'œuvre du compositeur György Ligeti qui, pour diverses raisons, n'avait pas abouti à la publication. Bayer et moi nous sommes donc rencontrés plusieurs fois chez lui et avons échangé avec passion au sujet de Ligeti, compositeur phare du XX $\mathrm{XX}^{\mathrm{e}}$ siècle. Hélas, Bayer devait succomber en 2004 à la longue maladie qui l'affectait. N'ignorant pas son état de santé, il me confia, peu de temps avant de mourir, de précieux documents sur Ligeti, notamment des entretiens dont un extrait est rédigé ici.

Ces entretiens ont été enregistrés en français, à Paris puis à Hambourg, entre le 23 novembre 1984 et le 10 juin 1985. Cet enregistrement comprend 7 cassettes audio, chacune d'une durée de 60 à $90 \mathrm{mn}$. Ce document est demeuré inédit à ce jour.

Quelques précisions quant à la transcription de cet enregistrement : les deux musiciens y évoquent souvent des œuvres de jeunesse de Ligeti, dont certaines sont inachevées ou ne sont pas publiées. Francis Bayer se réfère à la numérotation 
établie dans le catalogue d'Ove Nordwall, le premier musicologue ayant tenté d'établir une liste des œuvres de Ligeti. Pour faciliter la lecture, nous avons écrit dans le présent texte directement le nom de l'œuvre de Ligeti auquel le numéro se réfère dans ce catalogue. Par ailleurs, signalons le travail remarquable de Heidy Zimmermann, paru récemment et qui dresse une liste des œuvres du compositeur à partir des manuscrits conservés (Inventare der Paul Sacher Stiftung $n^{\circ} 34$, Sammlung György Ligeti, Musikmanuskripte, Bâle, Schott, 2016).

\section{Paris, le 23 novembre 1984.}

György Ligeti : Il existe jusqu'à maintenant seulement le livre de Nordwall'1 , qui est très bien, surtout en suédois (la version allemande n'est pas très bien traduite). Il y a aussi un petit livre anglais, de Griffiths ${ }^{2}$, qui est très juste, très bien, mais très populaire 3 . Connaissez-vous les entretiens?

\section{Francis Bayer : Oui.}

G. L. : Je dois vous informer qu'il y a encore Pierre $\mathrm{Michel}^{4} \ldots$

F. B. : Je ne le connais que de nom. Je n'ai pas eu l'occasion de le rencontrer, parce qu'il est à Strasbourg.

G. L. : C'est un jeune musicologue français, plus jeune que vous, qui a fait les traductions de mes articles pour les éditions Bourgois ${ }^{5}$. Il a écrit un livre sur moi où il y a aussi un très long entretien - nous avons eu de longues conversations pendant plusieurs jours - et aussi des analyses. Il est en train de le publier. En tout cas je crois que ce sera quelque chose qui va dans une direction très différente de la vôtre. Ce que j'ai vu de votre livre est vraiment d'un très haut niveau. Ce n'est pas une critique contre Michel...

F. B. : Son livre est davantage une introduction.

G. L. : Oui, il contient également des analyses très concrètes, mais pas un degré de réflexion aussi abouti que, par exemple, dans votre livre sur l'espace sonore ${ }^{6}$. Il existe encore autre chose, du journaliste musical Jean-Noël von der Weid ${ }^{7}$, qui veut faire un chapitre sur moi dans un livre (qui devrait paraître l'an prochain) où il y a différentes choses, littérature, photos, cinéma... Mais c'est plutôt littéraire, poétique, et pas scientifique. Je vous dis ça pour que vous sachiez qu'il y a des projets en France - j'aime donner ces informations avant -, mais c'est vous qui faites pour la science. 
F. B. : Je fais quelque chose que j'espère le mieux possible, et le plus complet possible, mais ça n'empêche pas d'autres personnes de travailler sur votre musique, au contraire.

Je voulais surtout vous poser des questions aujourd'hui sur la première partie de votre vie, c'est-à-dire avant que vous ne passiez en Occident. J'ai lu, je crois, à peu près tout ce qu'on peut lire, c'est-à-dire les textes que vous avez vous même écrits, dans Mein Judentum ${ }^{8}$...

G. L. : Oui, c'est un peu unilatéral ; on m'avait demandé quelle était ma relation avec la religion juive. C'était pour une série de conférences radiophoniques (j'ai seulement écrit cet article et il a été lu sur une radio de Stuttgart). On m'avait demandé de parler seulement de cet aspect. Je suis juif mais ça ne joue qu'un très petit rôle pour moi. L'oppression d'Hitler a joué un rôle important, mais nous n'étions pas une famille religieuse. À cause de cet article, on a cru que c'était une chose primordiale pour moi, mais ce n'est pas le cas.

F. B. : Si on lit votre article Mein Judentum, et l'autre article qui s'appelle Errinerung aus mein Kinheit ${ }^{9} .$.

G. L. : ...musikalische. Cet article contient seulement des renseignements sur la musique. C'est un autre aspect.

F. B. : En dehors de ces deux aspects, l'aspect de votre relation avec les Juifs et l'aspect d'enfance musicale de futur compositeur, que faudrait-il ajouter pour avoir une image plus complète de votre enfance?

G. L. : Je voudrais dire d'abord que ces deux aspects sont une image très mince. Il y a beaucoup d'autres choses, mais il m'est difficile d'en parler. D'abord, devenir musicien plus tard n'était absolument pas prévu - et je l'ai d'ailleurs dit dans cet article, mais j'y ai seulement retenu les aspects musicaux (que l'on comptait dans la famille un célèbre violoniste etc.).

La vie, mon enfance, était tout à fait différente de ces deux aspects. D'abord, par la culture, dans cette petite ville de Dicsőszentmárton, en Transylvanie, dont mes parents n'étaient pas originaires, puisqu'ils venaient de Hongrie. Ils se sont mariés à Budapest, mais mon père ${ }^{10}$ était de la région du lac Balaton. Et ma mère ${ }^{11}$ aussi était originaire d'une famille de la région de ce lac. Elle n'avait jamais vécu à Budapest, au contraire du reste de la famille, tous mes oncles et tantes. Une de mes tantes, qui a joué un très grand rôle dans ma vie, est venue avec nous dans la petite ville de Dicsőszentmárton, à cause de mon père, et s’y est mariée. 
F. B. : S'agit-il de la tante Marcsi ?

G. L. : Oui, Marcsi. Elle était presque comme ma mère. La ville était petite ; on était toujours très proche. C'était une sœur de mon père, plus jeune, presque du même âge que ma mère, un personnage des plus importants pour moi.

La famille était presque entièrement à Budapest, ce qui veut dire que mes parents étaient comme des étrangers en Transylvanie. C'était aussi une question de langue parce que tout le monde parlait roumain, les hongrois aussi, mais mes parents non. Ce qui était plus important pour moi que la culture juive, que je connaissais très peu, était d'être en Roumanie avec une langue qui était celle de la minorité. J'ai eu comme ça une éducation littéraire, tout un background culturel vraiment hongrois. Petit, je parlais aussi parfaitement l'allemand car j'avais une femme de ménage originaire de Vienne. (Et comme je n'ai pas parlé allemand ensuite pendant des années, j'ai dû réapprendre... j'avais oublié.)

Ce qui a joué un très grand rôle pour moi, et aussi dans ma musique, c'est une certaine ambiance de la culture hongroise, qu'il est très difficile de s'imaginer quand on n'est pas hongrois. C'est une langue tellement différente, avec une pensée et des structures tout à fait spéciales. Quand j'étais enfant, ça n'était pas conscient naturellement, mais ça l'était par exemple quand j'étais au lycée ${ }^{12}$. J'étais au lycée roumain, mais j'ai passé le baccalauréat dans un lycée hongrois, j'avais changé. Plus tard, les particularités de la grammaire et de la syntaxe hongroises sont devenues très importantes pour moi. Je suis très lié aussi à la poésie hongroise, plus encore à la langue qu'à la musique populaire.

Pourtant, j'ai été familiarisé à la musique populaire tout petit ; on entendait beaucoup de mélodies, surtout en Transylvanie. La musique était un autre aspect important, mais j'ai déjà mentionné ça. La musique officielle était la musique tsigane, parce qu'il y avait beaucoup de tsiganes dans la petite ville de Dicsőszentmárton. Je n'aimais pas cette musique. Mais il y avait aussi ce que Bartók et Kodály avaient découvert, le vrai chant paysan hongrois, encore très vivant (et qui d'ailleurs n'existait plus à Budapest). Entre autres, c'était notre cuisinière hongroise qui chantait ça.

Mais mon éducation musicale était faite surtout des disques que l'on possédait, on n'avait pas de piano. J'écoutais des choses plutôt tragiques, des opéras etc. Notre entourage musical était tout à fait provincial.

Dès neuf ans, et plus tard, aussi, au lycée, j'ai eu une orientation très unilatérale, très orientée vers les sciences naturelles. J'étais bon en mathématiques, en physique, chimie. En revanche, j'étais très mauvais en langue, en histoire. Et après, ça a changé, c'est curieux. Par exemple, aujourd'hui, je parle assez bien le français, mais quand j'étais au lycée, dans ma cinquième classe, j'ai dû repasser mon examen de français. Je détestais l'histoire. Aujourd'hui, et depuis plus de trente ans, j'ai une orientation beaucoup plus large : les sciences m'intéressent 
toujours, mais aussi les sciences humaines, pas seulement les sciences naturelles. Je suis très intéressé par les livres sur la culture et l'histoire. Au lycée, j'étais rigoureusement rationaliste. Ça venait sûrement de mon père, qui était ce type d'athée rationaliste du XIX ${ }^{\mathrm{e}}$ siècle, d'orientation politique très à gauche (sans être communiste, ni même socialiste, il n'adhérait à aucun parti). Moi, j'étais de son avis (mais aujourd'hui, je ne suis absolument pas de gauche).

Je vivais en Transylvanie dans une ambiance d'intellectuels de culture hongroise, très différente d'ailleurs, à Cluj, de quand j'étais tout petit. C'était une ville entre cent et cent cinquante mille habitants, la plus grande ville [de la région], mais quand même très provinciale. Cette double culture, hongroise et roumaine, que j'ai mentionnée dans l'article sur la musique, était une donnée très intéressante. La majorité était hongroise, parlait hongrois (alors qu'aujourd'hui non, c'est tout à fait roumain). Mais j'ai eu des amis roumains, quand j'étais au lycée roumain, et comme ça je suis entré un peu dans cette ambiance tout à fait différente des intellectuels roumains, moins nombreux. La Hongrie avait une orientation d'abord autrichienne, allemande, et une culture beaucoup plus répandue, avec quantité d'hommes de lettres, d'intellectuels. Alors qu'en Roumanie, beaucoup moins, c'était beaucoup plus primitif, un pays tout à fait agricole (La Hongrie l'était aussi, mais moins que la Roumanie). Il y avait une couche très mince d'intellectuels, mais cette couche, cette petite minorité était excellente, à Bucarest mais aussi à Cluj. J'ai eu des professeurs au lycée, surtout en mathématiques, qui était vraiment d'un très haut niveau ${ }^{13}$ (je peux le dire en connaissant beaucoup d'hommes de sciences aujourd'hui).

La Roumanie était axée sur la culture française. J'ai adopté comme ça un peu l'orientation hongroise, la culture germanique, mais aussi la culture et la littérature françaises. Au lycée on devait lire Pascal, de petits extraits... ou des gens d'Église comme Bossuet. Des choses très spéciales, même en français, c'est spécial [rires]. Par contre, ça s'arrêtait au XIX ${ }^{\mathrm{e}}$ siècle. Même Verlaine, Rimbaud et Baudelaire n'étaient pas enseignés... c'était déjà trop maudit [rires]. On lisait la littérature ancienne, différents poètes mineurs de la Pléiade, et beaucoup d'autres choses, naturellement beaucoup Molière, Racine, Corneille. On nous enseignait surtout la littérature, pas la musique ni la peinture. C'était une vie très isolée, et je connaissais la peinture seulement à travers les livres. On avait très peu la possibilité de voyager ; Le plus loin où nous puissions nous rendre était Budapest et Bucarest. Enfant, et jusqu'au lycée, je ne suis jamais allé dans d'autres pays que la Hongrie et la Roumanie. On voyageait très peu.

F. B. : À propos de la peinture, puisque vous parlez de ces images que vous voyiez sur les livres, vous avez écrit un chœur, quand vous étiez encore à Cluj, qui s'appelle Funérailles en mer [Temetés a tengeren]. Est-ce que ça a un rapport avec le tableau de Turner? 
G. L. : Non. Je ne connais pas ce tableau, même aujourd'hui.

F. B. : C'est un tableau qui se trouve à Londres...

G. L. : J'aime beaucoup Turner, mais il n'y a aucun rapport. J'ai écrit ce chœur à partir d'un poème d'Endre Ady, un poète très connu, qui a vécu d'ailleurs longtemps ici à Paris mais qui écrivait seulement en hongrois. Il est considéré comme un grand poète (c'est l'un des premiers hongrois de ce rang), mais, pour moi, il ne l'est pas vraiment. Naturellement, comme quelques jeunes, j'étais très attiré par sa poésie, symboliste, très pathétique. Funérailles est basé sur un de ses poèmes, peut-être était-ce lui qui connaissait le tableau de Turner? Mais je ne crois pas. Il connaissait la Bretagne, ce poème décrit les funérailles de pêcheurs bretons noyés. Peut-être qu'il a vécu cette scène lui-même quand il s'y trouvait ? C'est un poème qui m'a beaucoup touché : les pêcheurs ont de très grandes bottes, et, en cas de tempête, si le bateau vient à se renverser, ils meurent parce que leurs bottes sont trop lourdes. Mais ils doivent les porter pour l'équilibre. C'est un grand poème tragique qui a pour sujet la mort... avec des cadavres (La partition est à Vienne, pas à Hambourg ${ }^{14}$ ).

Mais c'est un exemple de l'ambiance, de la littérature hongroise. J'ai su lire très jeune, à trois ans, et à quatre ans déjà j'avalais les livres. C'était surtout des contes, les contes des Mille et une nuits (l'édition pour les enfants, en hongrois) et tous les contes allemands, de Grimm et autres, puis aussi Andersen et beaucoup de contes populaires hongrois (il existait une très belle collection, l'équivalent de Grimm en allemand ou Perrault en français, contes de la fin du XIX siècle). Et j'ai lu dès cinq ans des romans pour adultes, qui n'étaient pas bien pour les enfants, peut-être que je l'ai mentionné...

\section{F. B. : Krúdy?}

G. L. : Krúdy ${ }^{15}$, oui, que j'ai lu à cinq ans. Cet auteur n'était pas permis pour les enfants, parce que c'est très mélancolique, dépressif, nostalgique. C'est un grand écrivain, je crois qu'il m'a beaucoup marqué.

Que puis-je dire encore de ce temps-là ? Un odieux climat politique est apparu : il y avait d'abord les querelles entre Hongrois et Roumains, puis l'antisémitisme, et tout s'est superposé. Mais ce n'était pas durant mes dix premières années, c'était après.

J'ai eu une enfance assez protégée dans une famille entre la petite et la moyenne bourgeoisie, famille d'intellectuels mais pas riche, un peu restreinte financièrement. On a toujours vécu très simplement bien que ma mère fût médecin (elle a vécu très longtemps, elle est morte il y a seulement deux ans), ophtalmologue, et mon père... mais ça je l'ai écrit... 
F. B. : Oui, vous avez beaucoup parlé de votre père, mais, ce que je ne savais pas, c'est si votre mère exerçait son métier de médecin.

G. L. : Oui, mais pas forcément pour gagner de l'argent. Elle avait de ce point de vue très bon cœur et presque personne ne payait. Mon père avait une bonne situation (ça s'est dégradé par la suite). On n'a jamais été pauvre, je ne peux pas dire que j'ai vécu mon enfance dans la pauvreté : avec quelques restrictions mais jamais sans grands problèmes. C'était une façon de vivre, toujours au même endroit, voyageant très peu (il ne nous était pas possible financièrement de partir en vacances l'été).

Quand nous sommes allés vivre à Cluj, ma tante Marcsi et mon oncle, qui s'appelait Henrik, sont restés à Dicsőszentmárton. Puis ils se sont installés dans une autre ville de Transylvanie qui s'appelle Târgu Mureş en roumain (Marosvásárhely en hongrois), une petite ville de cinquante mille habitants à l'époque, traversée par le grand fleuve Mureş. C'est dans cette autre ville que j'ai passé presque tous mes étés pendant des années, chez ma tante. C'était une ville qui était importante pour la tradition hongroise, parce qu'elle appartenait à cette région que l'on appelle des Sicules (des Hongrois qui parlent un dialecte). C'était une région un peu spéciale, la seule qui, à l'époque médiévale, n'ait pas connu le féodalisme. Peuplée de Sicules (de traditions très différentes), et d'Allemands (que l'on nomme Saxons en Transylvanie, même s'ils ne sont pas saxons ; leur langue est proche de celle que l'on parle au Luxembourg et dans la région de Haute-Moselle, un dialecte franc, comme l'ancien français), ses habitants bénéficiaient de certains privilèges. Les rois hongrois du XII ${ }^{\mathrm{e}}$ siècle avaient laissé la liberté à ces paysans, ils n'étaient pas asservis, en échange du poste de garde-frontière qu'ils occupaient ; c'était des militaires. Ces avantages sont restés, et, à cause de ça, la culture sicule avait quelque chose de tout à fait spéciale... c'était des gens très fiers, voire vaniteux, orgueilleux. L'ambiance entre les deux villes, Cluj qui était majoritairement hongroise (mais hongroise « normale »), et Târgu Mureş était particulière. On y comptait de très anciennes écoles. On ne les nommait pas universités, mais simplement écoles (et elles sont devenues lycées plus tard). C'était des écoles supérieures, de théologie par exemple... Peut-être connaissez-vous le nom Bolyai, c'était un mathématicien?

\section{F. B. : Oui !}

G. L. : Il en existe deux. Farkas ${ }^{16}$ le père, et János ${ }^{17}$, son fils. János Bolyai était l'un des inventeurs de la géométrie non euclidienne (avec Lobatchevsky ${ }^{18}$ ). C'était au début du XIX ${ }^{\mathrm{e}}$ siècle $^{19}$. Il a vécu dans cette ville. Târgu Mureş était marquée d'une tradition culturelle plus forte encore qu'à Cluji ${ }^{20}$. Et ça m'a peutêtre marqué aussi. 
J'étais un enfant difficile, très névrotique, avec d'énormes phobies, des peurs terribles, toujours malade (mais jamais très gravement). J'ai été immobilisé comme ça pendant plus d'un an à cause d'un accident, une petite chose de rien au départ qui s'est transformée en une inflammation de l'articulation du genou. J'étais tombé d'un rocher, et on ne l'a pas soigné. Entre huit et neuf ans - une très mauvaise période - j'ai dû rester au lit, allongé pendant un an et demi. Après quoi, j'ai dû réapprendre à marcher; c'était très curieux, très difficile. C'est peut-être à cause de ça que j'ai lu beaucoup, puisque j'étais immobilisé. Ce n'était pas une maladie grave, mais une maladie qui a tout changé. Car j'étais avant comme tous les petits garçons. Et la volonté de devenir compositeur remonte peut-être à cette époque... volonté en tout cas d'avoir une orientation très intellectuelle. Mais j'ai toujours pensé devenir scientifique, et la musique m'est venue très tard malheureusement.

F. B. : Au point de vue de la musique, je voulais vous poser une question très précise : vous avez commencé à l'âge de quatorze ans [en 1937] à faire du piano en jouant des pièces assez simples, pour débutant, et vous avez immédiatement commencé à composer des pièces dans le style de Grieg. Quand avez-vous connu ou joué les pièces de Bartók du Mikrokosmos?

G. L. : D'abord Mikrokosmos n'existait pas. Je l'ai connu seulement après la guerre. Bartók l'a composé pendant les années 1930 mais ça n'a été publié que dans les années 1940 (chez Boosey \& Hawkes en Amérique). Mais c'était déjà la guerre. $[\ldots]^{21}$ Bartók était le plus grand compositeur, mais quand il était encore en Hongrie (il avait émigré en 1940 et il n'est jamais revenu car il est mort en 1945), je ne l'ai jamais vu personnellement, même s'il est venu à Cluj deux fois pour des concerts. Notre famille allait parfois aux concerts mais nous ne suivions pas la vie musicale ; c'était un coin de l'Europe très isolé... dans les montagnes. Bartók était quelqu'un de très indépendant, intransigeant, et il n'était pas aimé par la vie culturelle officielle en Hongrie. Il était aussi d'orientation politique de gauche, ce qui n'était pas bien vu sous la politique de droite pro-italienne. D'ailleurs il n'a pas voulu rester dans un pays qui était allié d'Hitler. Sa musique était très peu jouée, tant à la radio qu'en concert. Pourtant, c'était un grand pianiste et il jouait souvent à Budapest les œuvres du répertoire. Aujourd'hui on croit que sa musique était déjà réputée, mais non! Dohnányi ${ }^{22}$ comptait beaucoup ; c'était un personnage important mais pas comparable à Bartók. Et même Kodály était beaucoup plus connu. Bartók était un moderniste. Je n'ai pratiquement pas entendu sa musique à la radio, même si son nom était connu. Je crois n'avoir entendu Bartók qu'à dix-sept ou dixhuit ans, jamais avant. Et dans mes leçons de piano, Bartók n'existait pas.

F. B. : Je voulais justement en venir à certaines de vos œuvres que vous avez composées à cette époque-là, quand vous étiez à Cluj avant la guerre. Je sais que 
certaines sont perdues, j'ai des photocopies d'autres, que monsieur Nordwall m'a faites. Par exemple, je voulais vous posez une question à propos d'une série de quatre petites pièces pour piano, qui sont toutes avec un ostinato...

G. L. : Oui, c'était en 1941, j'étais déjà dans la classe de composition.

F. B. : Est-ce que le titre Basso ostinato est pour l'ensemble des pièces?

G. L. : Oui. Elles sont toutes sur basse obstinée. Je crois en fait que j'ai fait mon examen d'admission dans la classe de composition avec ça. En 1941, j'ai passé mon baccalauréat et en septembre, j'ai passé l'examen pour entrer au conservatoire.

F. B. : Est-ce qu'il y a dans les idées musicales de ces quatre pièces des réminiscences de chants populaires hongrois, ou c'est entièrement de votre invention?

G. L. : C'est dans le style... Peut-être qu'ici, il y a un modèle de chant d'enfant hongrois? [chantant] En fait non! C'est en mode phrygien, ça n'existe pas [chantant de nouveau]. Il y a un modèle mais ça n'existe pas comme tel... La deuxième pièce est [fondée] sur [l'intervalle de] triton : non!

F. B. : À propos de cette seconde pièce : Dans votre conversation avec Péter Várnai ${ }^{23}$, vous parlez de ce qui vous a amené à écrire de la musique de style mécanique, et vous dites que cela ne date pas du Poème symphonique pour cent métronomes ou de la section des Nouvelles Aventures qui s'appelle Les Horloges démoniaques, mais que, quand vous étiez jeune, vous avez écrit une pièce où la main gauche joue une progression mécanique de triton pendant que la main droite joue également quelque chose qui est mécanique. S'agissait-il de cette pièce?

G. L. : Oui, mais aussi d'une autre pièce pour piano à quatre mains, encore plus mécanique. Les tritons sont ici, c'est ça.

F. B. : Vous parliez aussi précisément de cette pièce à quatre mains où il y a quatre ostinatos différents... [Il s'agit de Tréfás Induló (Marche amusante), allegro pour piano à quatre mains de 1942]

G. L. : Cette pièce est un peu comme la première des trois pièces pour quatuor de Stravinsky, ce modèle qui se répète. Mais je ne connaissais pas Stravinsky. Je crois qu'il y a plusieurs pièces machinales. J'ai obtenu la partition de cette marche [Tréfás Induló] par Nordwall. Je crois que ce sont des choses qu'il a 
trouvées récemment à Budapest. Vous avez d'autres pièces pour piano à quatre mains?

F. B. : Non, c'est la seule.

G. L. : Il en existe une quantité. Je vais les chercher pour vous, et vous m'envoyez en échange les quatre pièces sur basse obstinée. J'ai été très étonné parce que je me souvenais que j'avais fait ça, mais... Je me demande si dans la troisième... [chantant] Là, il y a un modèle de chant populaire hongrois! Mais je crois que c'est seulement un modèle, ce ne sont pas vraiment des chants populaires. En ce temps-là, je connaissais naturellement déjà beaucoup la musique de Bartók. Mikrokosmos n'était pas prêt (en tout cas pas publié), mais les pièces comme Pour les enfants ou beaucoup d'autres... À dix-huit ans [en 1941], je connaissais déjà bien l'œuvre de Bartók.

F. B. : Toujours au sujet des œuvres de cette période (les années 1940), il y a un Trio pour piano, violon et violoncelle...

G. L. : Oui, ça existe. Ce fut ma première pièce jouée.

F. B. : Est-ce que vous jouiez vous-même lors de l'exécution?

G. L. : Oui, avec deux amis.

F. B. : La partie de piano?

G. L. : Oui. C'est une pièce très primitive.

F. B. : Elle est pleine de charme, et l'on voit déjà l'écriture en canon dans l'introduction lente.

G. L. : Je crois que j'étais déjà dans la classe de composition. Les pièces sur ostinatos sont les dernières que $\mathrm{j}$ 'ai faites tout à fait naïvement. Après, $\mathrm{j}$ 'ai eu un très grand professeur : Ferenc Farkas.

F. B. : Je crois que j'ai entendu une fois une pièce de lui qui était une transcription pour orchestre à cordes de certaines danses.

G. L. : Il en a fait beaucoup, oui. Comme compositeur, il n'est pas très important, mais c'était un professeur génial, très sévère. En une année scolaire, il m'a fait apprendre toute l'harmonie classique. On avait fini avec l'harmonisation dans le 
style de Bach, les modulations... Puis une année supplémentaire pour l'harmonie sur une basse... ça marchait très vite parce qu'il était très exigeant. J'ai fait une quantité d'exercices d'harmonie, de contrepoint. C'était très rodé chez lui, et grâce à ça j'ai avancé rapidement en ce temps-là... aussi parce que j'étais tout à fait naïf quand je suis entré dans la classe de composition.

F. B. : Est-ce qu'il reste des traces de la Symphonie que vous aviez entreprise en 1938 ?

G. L. : Non. C'est dommage, ce serait un document d'une naïveté très touchante. Je me rappelle où je l'ai laissé dans notre appartement [à Cluj]. Quand mes parents et mon frère ont été déporté à Auschwitz, et que je suis allé au service du travail (militaire), les meubles que nous avions ont été tous emportés, on ne les a jamais retrouvés. Plus tard, quand ma mère et moi sommes revenus (mon père et mon frère étaient morts), on nous a redonné le même appartement, mais on a dû chercher d'autres meubles. C'était un appartement de trois pièces, une pour mon père, une pour ma mère et une pour les deux enfants. C'était assez restreint et ma mère donnait ses consultations d'ophtalmologie dans sa pièce. Malgré cela, et ce fut très important pour mon enfance, mon père avait une très grande bibliothèque (j'étais très fier qu'il ait plus de trois mille livres). Mais après, quand ça a marché moins bien pour nous - on avait toujours eu des appartements d'environ quatre pièces -, toute la bibliothèque est passée à la cave, il restait très peu de livres dans l'appartement, c'était vraiment tragique. Mais il a toujours cru qu'Hitler allait perdre la guerre et qu'après nous pourrions commencer une autre vie; ça a commencé pour moi, mais pas pour lui. Dans la pièce que nous partagions avec mon frère, il y avait une grande table (divisée en deux, pour chacun des deux enfants) dans laquelle il y avait une sorte de tiroir où l'on pouvait mettre des documents. Tout est resté dedans. La Symphonie était dans un dossier ; tout a disparu. Ce qui est resté, comme ces partitions, est le produit du hasard... je ne sais pas comment.

F. B. : Oui, il y a aussi des extraits d'un quatuor à cordes, qui n'est pas complet.

G. L. : Oui, un mouvement je crois.

F. B. : Sonatina mi-minore pour quatuor à cordes. Il y a quand même six pages.

G. L. : Il y a quelque chose qui était resté quand nous sommes revenus, moi de la guerre et ma mère des camps de concentration. On a retrouvé des choses dans la cave, des vêtements, des partitions et aussi des livres. J'ai encore aujourd'hui des livres qui étaient chez mes parents quand j'étais petit. Je crois qu'ils avaient 
eu le temps de mettre certaines choses dans de grandes caisses de bois, il y avait pas mal de place à la cave. C'est d'ailleurs ce qui est resté. Je n'étais plus chez moi, j'étais déjà militaire depuis deux mois. Je crois qu'il n'y a aucun espoir de retrouver aujourd'hui ce qui n'était pas à la cave. C'est dommage, j'ai tout oublié de cette Symphonie, j'aimerais tellement. J'ai même oublié les thèmes, je ne sais pas pourquoi... J'avais fait seulement deux mouvements...

F. B. : C'était donc une symphonie inachevée...

G. L. : Très inachevée... [rires] et aussi totalement naïve, parce que ce que j'avais lu dans ce livre d'orchestration ${ }^{24} \ldots$ c'était tout à fait impossible.

F. B. : Je sais qu'après la guerre vous êtes rentré chez vous à pied à partir d'Arad...

G. L. : Non, c'était près de Debrecen, dans une forêt au sud. Je n'y suis jamais retourné jusqu'à aujourd'hui. Entre Debrecen et Oradea [Nágyvárad]. Heureusement qu'il y avait cette forêt parce que la plaine hongroise est totalement agricole et comme c'était en octobre, il était impossible de se cacher dans l'herbe (alors que l'été on peut se cacher dans les blés) : c'était une grande chance de trouver cette forêt ${ }^{25}$.

F. B. : Donc vous êtes revenus à pied à Cluj. C'était au mois de novembre?

G. L. : Non, c'était en octobre. Si je me souviens bien, je me suis caché le 10 ou le 11 octobre, et un jour après les Russes étaient là. Ensuite ça m'a pris du temps de retourner à Cluj à pied, par petites étapes, peut-être dix jours (on peut le faire en quatre jours, mais il y a quand même presque deux cents kilomètres). Je suis resté à Oradea quelques jours... j'étais à Cluj à la fin octobre, à peu près le 22 .

F. B. : Entre octobre 1944 et septembre 1945, vous avez passé l'année à Cluj.

G. L. : Oui, et en septembre 1945, je suis allé à Budapest.

F. B. : Et vous avez repris votre travail de compositeur...

G. L. : Oui. J'étais inscrit à l'université, qui avait rouvert très tôt. Les Russes étaient là en octobre, et déjà en novembre ou décembre on pouvait aller à l'université. Mais je ne suis jamais allé en cours. J'étais inscrit en philosophie et histoire de l'art, car c'était le plus simple et la peinture m'intéressait. Mais finalement je crois n'être allé qu'une fois en cours. Mais c'était important, pour manger, car il 
était possible d'aller au restaurant universitaire, c'était gratuit. On devait avoir un certificat d'étudiant, ou sinon, on devait aller faire des travaux pour les Russes. Et comme j'étais sans argent... J'ai travaillé aussi quelques semaines dans une librairie comme vendeur. Je ne sais pas comment j'ai vécu, on n'avait pas d'argent, les bourses n'existaient pas. Il existait une aide pour les personnes qui avaient été opprimés par Hitler; c'était une petite aide financière pour les premiers mois. Puis des amis m'ont aidé, quand j'étais étudiant - on en avait beaucoup -, alors j'ai pu me consacrer à la composition. J'ai trouvé un piano que j'ai loué pour quelques mois... c'était très compliqué.

J'ai aussi été malade une grande partie de ce temps-là. J'étais dans un hôpital, j'avais une pleurite. Ce devait être une infection que j'avais contractée quand j'étais militaire, au contact de la tuberculose. En décembre 1944, j'ai été immobilisé à l'hôpital trois mois ; on était très bien traité, à l'hôpital, sans payer.

F. B. : Et votre mère n'est rentré à Cluj que vers le mois de février ou mars...

G. L. : Avril, au début du mois d'avril 1945. Je me rappelle qu'entre décembre et février, j'étais malade, presque toujours à l'hôpital, elle est revenue et peu de temps après je suis sorti de l'hôpital.

F. B. : À ce moment-là, vous avez écrit un certain nombre d'œuvres, et notamment monsieur Nordwall m'a fait parvenir les photocopies d'un petit carnet noir sur lequel il y a des chants qui s'appellent Bicinia Bicice.

G. L. : Oui, c'est une chose très privée.

F. B. : Je crois en effet que c'est quelque chose de très personnel.

G. L. : Je n'ai pas de secrets [rires].

F. B. : C'est-à-dire qu'il y a une voix de baryton qui, je suppose, est la vôtre, et une voix de mezzo...[Bicinia biciae, sept duos composés entre le 7 juin et le 17 octobre 1945]

G. L. : Oui, j'avais une amie qui s'appelait Brigitte. Je l'ai épousée beaucoup plus tard, c'était mon premier mariage (Je me suis marié trois fois et je suis toujours marié aujourd'hui). Brigitte était une fille avec qui je chantais beaucoup dans des ensembles, des chorales, et aussi pendant un temps dans un ensemble professionnel (nous étions cinq et donnions des concerts à Cluj). On surnommait Brigitte " Bici », et comme il existait des pièces polyphoniques à deux voix appelées Bicinia, j’ai appelé ce recueil Bicinia biciae, pour Bici. 
F. B. : Je voulais vous poser la même question que pour les quatre petites pièces pour piano qui s'appellent Basso ostinato. Quand on joue au piano ces pièces, ou quand on les chante, on a l'impression qu'il y a quelque chose qui rappelle les chants populaires d'Europe centrale...

G. L. : Hongrois, pas généralement d'Europe centrale. J'ai été aussi beaucoup influencé par le folklore roumain, mais pas là.

F. B. : Est-ce qu'il y a véritablement des citations, des fragments, ou bien s'agit-il encore une fois seulement d'un climat?

G. L. : Il ne s'agit que d'un climat. Faites voir... ici est inscrit « Gyuri », c'est mon surnom (comme Bici). Je me demande si c'est mon écriture parce que Brigitte a beaucoup recopié ma musique aussi... Oui, c'est mon écriture (elle a beaucoup changé). Tout est un peu dans le style Bartók, Kodály, l'École hongroise. J'avais comme ça une grande quantité de cahiers noirs.

Cette pièce est sur un poème d'Attila József. Je l'aimais beaucoup ; j'en ai écrit aussi une version pour deux voix de femmes [Ligeti évoque ici Betlehemi Királyok].

[Puis, chantant la dernière pièce du cycle Bicinia biciae] Là, il y a un chant populaire, dans la dernière pièce [Repülj madár (Vole, oiseau !)], que j'ai arrangée pour deux voix, mais dans les autres, non. Je vois qu'il y a aussi un peu du caractère du folklore roumain, plutôt hongrois, mais avec un peu de roumain... ${ }^{26}$

\section{Notes}

1 Ove Nordwall, György Ligeti. Eine monographie, Mayence, Schott, 1971

2 Paul Griffith, György Ligeti, Londres, Robson Books, 1983

3 Ligeti entend par là que l'ouvrage est accessible à un public non expert.

4 Pierre Michel, György Ligeti, compositeur d'aujourd'hui, Paris, Minerve, 1985

5 Ce travail de traduction n'a pas été publié. Au final, c'est Catherine Fourcassié qui, des années plus tard, a traduit de l'allemand les articles pressentis : György Ligeti, Neuf essais sur la musique, Genève, Contrechamps, 2001

6 Francis Bayer, De Schönberg à Cage, Essai sur la notion d'espace sonore dans la musique contemporaine, Paris, Klincksieck, 1981

7 L'ouvrage, paru plus tard que prévu, est probablement : Jean-Noël von der Wied, La musique du XX $X^{\mathrm{e}}$ siècle, Paris, Hachette, 1992

8 « Mein Judentum », extrait de l'ouvrage réalisé par Hans Jürgen Schultz, Mein Judentum, Zürich, Düsseldorf, Benziger, 1999, pp. 209-221

9 « Musikalische Erinnerungen aus Kindheit und Jungend » [Souvenirs musicaux d'enfance et de jeunesse], à l'occasion du quatre-vingt-dixième anniversaire de Ludwig Strecker, Mayence, Schott, 1973, pp. 54-60

10 Sándor Ligeti, né Alexander Auer (1890-1945) 
11 Ilona Ligeti, née Ilona Somogyi (1893-1981)

12 Ici, Ligeti entend par lycée ce qui correspond en France au collège et au lycée réunis.

13 Dans d'autres textes, Ligeti cite notamment Márk Antal.

14 Comme beaucoup des manuscrits de Ligeti, la partition se trouve aujourd'hui au Paul Sacher Stiftung de Bâle, Suisse.

15 Gyula Krúdy (1878-1933)

16 Farkas Bolyai (1775-1856)

17 János Bolyai (1802-1860)

18 Nicolaï Ivanovitch Lobatchevsky (1792-1856)

19 Le système de Bolyai appelé « géométrie absolue » date de 1831.

20 Même si Ligeti emploie ici le nom allemand de Cluj, à savoir « Klausenburg ", nous avons préféré homogénéiser pour faciliter la lecture. Par ailleurs, Cluj s'appelle encore Kolozsvár en hongrois.

21 Fin de la face A, Cassette audio $n^{\circ} 1 / 7$

22 Ernő Dohnányi (1877-1960)

23 György Ligeti in conversation with Péter Várnai, Josef Häusler, Claude Samuel, and himself, Londres, Eulenburg, 1983

24 Sa tante Marcsi lui avait acheté le second volume du traité d'instrumentation d'Albert Siklós : Siklós Albert, Hangszereléstan, Második kötet, Budapest, Rozsnayi Károly, 1910

25 Le 23 septembre 1944, les premières unités de l'Armée Rouge franchirent la frontière hongroise. Le front se rapprochant, la forteresse d'Oradea, où Ligeti était détenu, fut évacuée. Les prisonniers furent transférés dans la basse plaine, à Berettyóújfalu, à quelques kilomètres des affrontements. Le 10 octobre, Ligeti et quelques-uns des détenus réussirent à s'évader du camp. Ils trouvèrent refuge dans un bois situé entre les villages de Konyár et Hencida, où ils attendirent l'arrivée des troupes soviétiques. Tous les autres détenus du camp furent déportés à Mauthausen.

26 Fin de la Cassette audio $n^{\circ} 1 / 7$ 